\title{
Text entry using soft keyboards
}

\author{
I. SCOTT MACKENZIE, SHAWN X. ZHANG and R. WILLIAM SOUKOREFF
}

Department of Computing and Information Science, University of Guelph, Guelph, Ontario, Canada N1G 2W1

\begin{abstract}
Text entry rates are explored for several variations of soft keyboards. We present a model to predict novice and expert entry rates and present an empirical test with 24 subjects. Six keyboards were examined: the Qwerty, ABC, Dvorak, Fitaly, JustType, and telephone. At 8-10 wpm, novice predictions are low for all layouts because the dominant factor is the visual scan time, rather than the movement time. Expert predictions are in the range of 22-56 wpm, although these were not tested empirically. In a quick, novice test with a representative phrase of text, subjects achieved rates of $20.2 \mathrm{wpm}$ (Qwerty), $10.7 \mathrm{wpm}$ (ABC), $8.5 \mathrm{wpm}$ (Dvorak), $8.0 \mathrm{wpm}$ (Fitaly), $7.0 \mathrm{wpm}$ (JustType), and $8.0 \mathrm{wpm}$ (telephone). The Qwerty rate of $20.2 \mathrm{wpm}$ is consistent with observations in other studies. The relatively high rate for Qwerty suggests that there is skill transfer from users' familiarity with desktop computers to the stylus tapping task.
\end{abstract}

\section{Introduction}

The rapidly expanding market for mobile computing systems has brought to the fore a difficult challenge: the need to manually enter alphanumeric information that is coded in machine-readable form, such as ASCII. Such systems include personal digital assistants (PDAs), personal information managers (PIMs), slates, and other pen-based products. Without a full-size physical keyboard, these systems resort to alternate techniques for text entry, such as handwriting recognition or tapping on a soft keyboard. Soft keyboards are keyboard images that appear on a computer's display and permit alphanumeric entry by directly tapping on them with a stylus. Typically, the display for a penbased system combines liquid-crystal output technology with a transparent overlaid pressure-sensing input technology. Thus, a stylus tap on a soft key is easily translated into the ASCII code for the character represented by the key.

This paper addresses the problem of text entry with a soft keyboard, and predicting and comparing the performance potential of alternate layouts for soft keyboards. We are particularly interested in the novice experience. Many mobile systems are targeted at the consumer market and, therefore, immediate ease of use is important. If the text entry method is cumbersome, slow, or error prone, user acceptance will be hindered.

\subsection{Mobile computing}

Since their introduction in the early 1990s, penbased systems have faltered in their user acceptance. Early systems suffered in the one area that garnered the most attention: handwriting recognition. Simply put, claims of unconstrained, cursive handwritten input did not meet the expectations of a demanding user community. Today, handwriting recognition products are much improved (Blickenstorfer 1996, 1997) and pen-based systems, particularly PDAs and PIMs, are alive and well. More important, however, is the shift in attention from the limited idea of 'penbased computing' to the more general notion of 'mobile computing'. The latter encompasses a vast territory, including not only PDAs, PIMs, slates, and other traditional pen-based products, but also cellular phones, pagers, remote controls for consumer products, or future 'convergent' products for interfacing to computing, cable, telephone, consumer electronics, and internet systems.

\subsection{Beyond Qwerty}

Mobile systems that support handwriting recognition generally include a soft keyboard as well. The soft keyboard is easy to implement and provides an alternative to handwriting. With physical keyboards, the idea of a non-Qwerty layout is a tired notion that is of minor interest today. Although alternate key layouts, such as Dvorak (Lewis et al. 1997), alphabetic (Norman and Fisher 1982), or chord keyboards (Gopher and Raij 1988), can potentially support higher entry rates, 
substantial practice is required to become proficient in their use. This, combined with the large installed base for Qwerty keyboards, has ensured the continued role of Qwerty as the keyboard of choice for desktop computing.

For soft keyboards, however, the arguments for using a Qwerty layout are diminished because touch typing skill will not necessarily transfer to 'touch tapping'. The motor skill of two-handed eyes-free touch typing is very different from the simple act of one-handed eyes-on tapping with a stylus on a soft keyboard. That is, if we compare two touch typists-one $25 \mathrm{wpm}$ and one $75 \mathrm{wpm}$ - in their ability to touch tap, it is not certain that the 75 wpm typist would be faster, or substantially faster, than the $25 \mathrm{wpm}$ typist.

\subsection{Predicting text entry speed on a soft keyboard}

In an earlier paper (Soukoreff and MacKenzie 1995), we described a comprehensive model to predict novice and expert text entry rates for stylus tapping on a soft keyboard. Since this model is important to the present discussion, we will briefly summarize it here. The model is comprised of five major components: (a) digitized layout of a keyboard, (b) Fitts' law for rapid aimed movements, (c) the Hick-Hyman law for choice selection time, (d) a linguistic table for the relative frequencies of letter pairs, or digraphs, in common English, and (e) a spreadsheet in which the preceding components are combined.

For (a), each key is assigned an $x-y$ coordinate, thus allowing digraph distances to be easily computed using the Pythagorean identity. The movement distance, or amplitude $(A)$, from key $i$ to key $j$ is

$$
A_{i j}=\sqrt{\left|x_{j}-x_{i}\right|^{2}+\left|y_{j}-y_{i}\right|^{2}}
$$

For (b), we use Fitts' law (Fitts 1954, MacKenzie 1992) to predict the movement time ( $M T$, in seconds) to tap any key given any previous key. This is a simple prediction based on the distance between the keys $\left(A_{i j}\right)$ and the size, or width, of the target key $\left(W_{j}\right)$ :

$$
M T_{i j}=0.204 \log _{2}\left(\frac{A_{i j}}{W_{j}}+1\right)
$$

For (c), we use the Hick-Hyman law (Hick 1952, Hyman 1953) to predict the reaction time ( $R T$, in seconds) in visually scanning a 27-key layout to find the target key. For novices, we set

$$
R T=0.200 \log _{2}(27)=0.951 \text { seconds }
$$

For experts, we set $R T=0 \mathrm{~s}$.
For (d), we use a $27 \times 27$ matrix of digraph counts to establish probabilities for each digraph in common English, $P_{i j}{ }^{1}$. These are used to weight the movement time predictions in obtaining the mean movement time over all possible digraphs:

$$
\overline{M T}=\sum_{i} \sum_{i}\left(P_{i j} \times M_{i j}\right)+R T
$$

$R T$ is set to either $0.951 \mathrm{~s}$ (novices) or $0 \mathrm{~s}$ (experts), as noted above.

Entry speed in words-per-minute (wpm) is calculated by taking the reciprocal of the mean movement time, multiplying by 60 seconds per minute, and dividing by 5 characters per word:

$$
\text { Entry_Speed }=\left(\frac{1}{\overline{M T}}\right) \times \frac{60}{5}
$$

Note that the definition of a word when computing typing speed is 'five characters including spaces' (Gentner 1983).

The model takes particular care to accommodate the space bar, since it is the most prevalent character in text entry tasks. The result is a general behavioural description and predictive model of the task of text entry with a stylus and soft keyboard. We consider the predictions approximate, but useful. For more details, see Soukoreff and MacKenzie (1995).

Our earlier paper developed the model in the context of the Qwerty layout only. Since then, we have incorporated some minor improvements and refinements, for example, in accommodating the space bar. Our predictions for the Qwerty layout stand as follows:

- Novice: 8.9 wpm

- Expert: $43.2 \mathrm{wpm}$

The novice prediction is difficult to validate empirically for two reasons. First, it is difficult to find subjects who have never used or seen a Qwerty keyboard and who, therefore, would exhibit visual scanning as predicted for novices. Second, in an empirical test the novice classification fades quickly since subjects gain familiarity with the key layout within a few taps. In fact, a real test of a novice prediction would require the keys to be randomly reassigned after each tap. ${ }^{2}$ So, we view the novice prediction as a lower threshold at which subjects begin to enter text, but from which they would rise quickly.

The expert prediction is also difficult to test for at least two reasons. First, proposing a single measure of expert performance is simplistic because users can always attain small improvements in performance, consistent with the power law of practice (De Jong 1957). Second, measuring expert performance requires a 
study conducted over many sessions, and this is very labour intensive. To our knowledge, no such study exists for text entry on a soft keyboard using a stylus.

There are a few studies using text entry with soft Qwerty keyboards, although the task was usually administered only for one or two hours. MacKenzie et al. (1995) reported rates of $22.9 \mathrm{wpm}$ for stylus tapping on a soft Qwerty keyboard. We expect that with practice entry rates would increase, leveling off near $43 \mathrm{wpm}$. Related studies used a soft keyboard on a touch screen with entry via the fingers rather than a stylus. Entry rates ranged from about $12 \mathrm{wpm}$ (Gould et al. 1990) to $25 \mathrm{wpm}$ (Sears 1991). In another study (Sears et al. (1993), subjects were allowed to use both hands on a touchscreen. Rates as high as $32 \mathrm{wpm}$ were recorded; however, the comparison with stylus tapping is weak.

Finally, it is important to note the limitations in the model. These are elaborated later in the context of an empirical test with several alternate layouts for soft keyboards.

\section{Soft keyboard layouts}

From the work described above, we proceeded to test our model on other soft keyboard layouts. Although there are numerous layouts to test, we limit our discussion to several interesting possibilities.

\subsection{Dvorak keyboard}

Since the Dvorak layout is well known as a Qwerty alternative, it was a logical starting point (see figure 1). In its physical form, the Dvorak keyboard is similar to a Qwerty keyboard. By renaming the keys, a Qwerty layout can be transformed into a Dvorak layout. The Dvorak keyboard was designed to optimize two-handed touch typing. The idea is that higher entry rates can be obtained if common digraphs are entered by fingers on opposing hands instead of on the same hand (Lewis et al. 1997). As well, the most common letters (e.g. E, T, A, $\mathrm{H})$ are positioned along the home (viz. middle) row.

After entering the $x-y$ coordinates of the Dvorak keyboard into our spreadsheet, the novice and expert predictions were immediately available to us. Our predictions are as follows:

- Novice: 8.7 wpm

- Expert: $38.7 \mathrm{wpm}$

At $38.7 \mathrm{wpm}$, the expert prediction is below our $43.2 \mathrm{wpm}$ prediction for a Qwerty layout. This illustrates the distinct difference between optimizing for two-

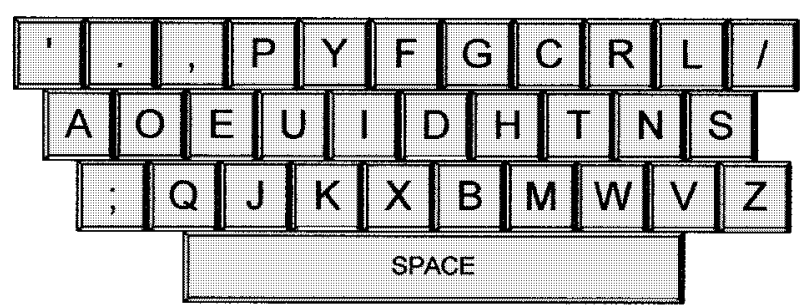

Figure 1. The Dvorak layout.

handed touch typing vs. optimizing for one-handed touch tapping with a stylus.

The novice rate of $8.7 \mathrm{wpm}$ is slightly lower than our Qwerty prediction of $8.9 \mathrm{wpm}$. In general, the novice prediction is dominated by the visual scan time, so any layout permutation that minimizes movement has only a minor impact on the novice entry rates. This point is emphasized in the following example: If we consider, in the extreme, that the novice visual scan time of $951 \mathrm{~ms}$ is the only component of the task, then this alone represents an entry rate of $(1 / 0.951) \times(60 /$ $5)=12.6 \mathrm{wpm}$. Therefore, novice predictions will always be lower than $12.6 \mathrm{wpm}$ by an amount determined by the movement component of the task.

\subsection{ABC layout}

In an effort to minimize screen real estate, soft keyboards can be streamlined, for example, by using short and wide or tall and narrow layouts. We investigated a variety of such possibilities. Figure 2 illustrates an example which we call the ABC layout.

There are certain advantages to grouping the keys as shown in figure 2 and by using a space bar that spans the full keyboard. The alphabetic ordering of keys gives novices a good indication of each key's location, and this should reduce the visual scan time. Since the space is the most prevalent character in text-entry tasks, the space bar's size and proximity to the other keys should reduce overall movement time.

After entering the key coordinates in our spreadsheet, the following predictions emerged:

- Novice: > $9.6 \mathrm{wpm}$

- Expert: $40.9 \mathrm{wpm}$

We indicate 'greater than' $9.6 \mathrm{wpm}$ for the novice prediction for the simple reason that the sequenced ordering of keys precludes anyone from being a novice, provided they know the alphabet (a reasonable assumption). 


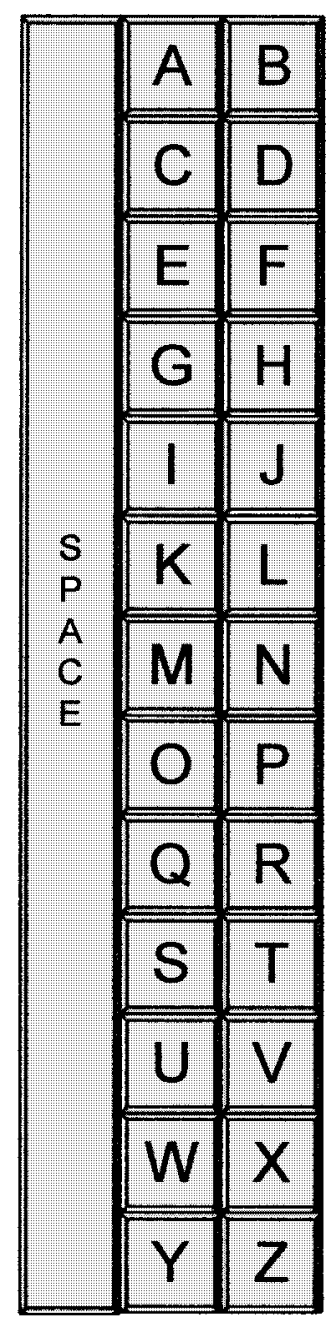

Figure 2. The ABC layout.

The expert prediction is slightly lower than our $43.2 \mathrm{wpm}$ prediction for the Qwerty layout. The close proximity of all keys to the space bar is a definite advantage for the ABC layout; however, this appears to be offset by placing letters in two columns. Words like 'satisfaction' require substantial up-down-up pen travel, and this tends to push the prediction down. A threecolumn version would alleviate this, but there is a cost, since shifting some keys to the third column increases their distances to the space bar. Such an arrangement was not tested.

\subsection{Fitaly keyboard}

The Fitaly soft keyboard is a commercial product ${ }^{3}$ designed to optimize text entry with a stylus (see figure 3). The most striking feature of the layout is the

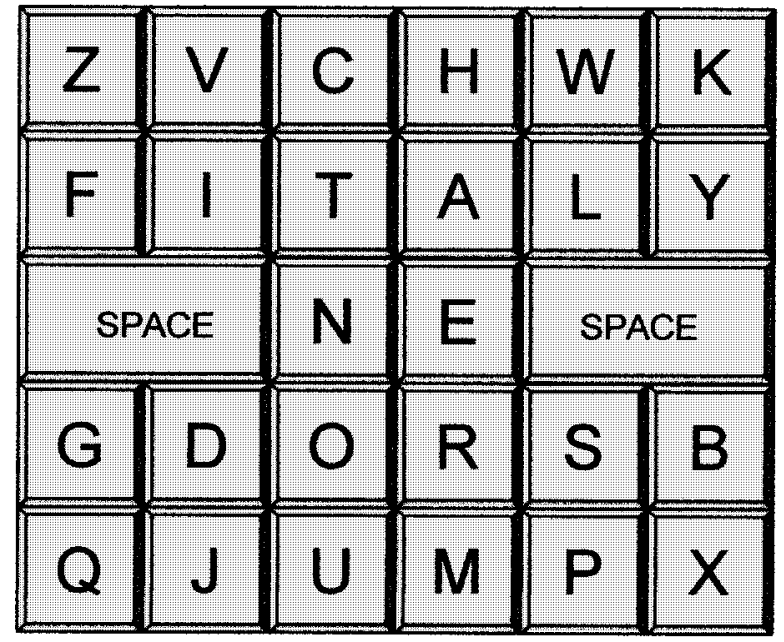

Figure 3. The Fitaly layout.

presence of two space bars. The proximity of the most common letters in English (e.g. E, T, A, N) to the space bar is also immediately apparent. The keyboard's name is taken from the letter sequence along the second row of keys.

The Fitaly keyboard was designed to minimize the travel from one letter to the next. According to the developer, the average travel is 1.8 keys compared to 3.2 keys for a Qwerty layout. These figures were obtained using a corpus of digraph probabilities similar to that described by Soukoreff and MacKenzie (1995).

After entering the $x-y$ coordinates of the Fitaly keys into our spreadsheet (and introducing a few modifications to accommodate two space bars), we obtained the following predictions:

- Novice: 9.7 wpm

- Expert: 55.9 wpm

The novice prediction at $9.7 \mathrm{wpm}$ is close to the predictions given earlier. The expert prediction of $55.9 \mathrm{wpm}$ is impressive. This is a full $24.8 \%$ higher than the $43.2 \mathrm{wpm}$ expert prediction for the Qwerty layout. To our knowledge, no empirical evaluation of the Fitaly soft keyboard exists.

\subsection{Telephone keyboard}

Although extensive text entry via a telephone keyboard is arguably impractical, some text entry occasionally occurs using this familiar product (see figure 4). Note that the letters $\mathrm{Q}$ and $\mathrm{Z}$ are not represented on the standard telephone keyboard. They are commonly assigned to the keys 7 and 9, respectively. 
Examples of text entry via a telephone include automated directory assistance, automated facsimile request (also known as 'fax back'), or programming names and numbers into home and cellular telephones. Since the telephone keyboard is small (12 keys), it is an appealing choice for other mobile products that require text entry. So, the idea of modeling and predicting the text entry task for the telephone keyboard is perhaps worthwhile. We have chosen two techniques to explore here.

2.4.1. With disambiguation: Since there are three or four letters assigned to each key, the telephone keyboard poses a special problem: disambiguating the input. One technique uses a dictionary and a built-in disambiguating algorithm to determine each word. This will work especially well if the dictionary is small, as, for example, in finding a phone extension in a small company by entering an employee's last name. Consider the following sequence:

$$
\begin{array}{lllll}
5 & 6 & 6 & 3 & 7
\end{array}
$$

Although this sequence has $3 \times 3 \times 3 \times 3 \times 4=324$ permutations, most are nonsense. If the dictionary includes only employees' last names, the most likely name is 'Jones', illustrated as follows and easily verify by examining figure 4 :

$$
\begin{array}{ccccc}
\mathrm{J} & \mathrm{O} & \mathrm{N} & \mathrm{E} & \mathrm{S} \\
5 & 6 & 6 & 3 & 7
\end{array}
$$

Our novice and expert predictions for the telephone keyboard with built-in disambiguation are as follows:

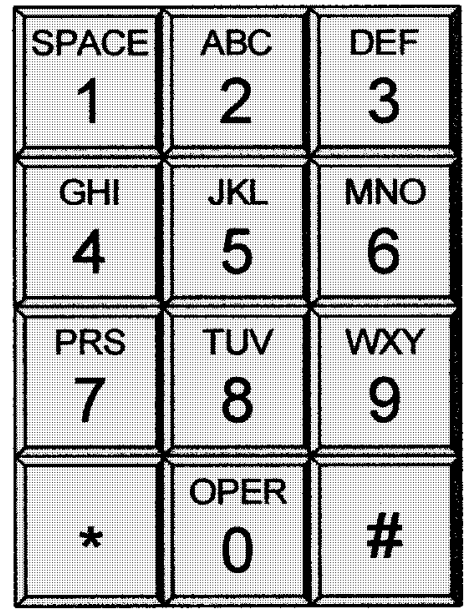

Figure 4. The telephone keyboard layout.
- Novice: > $9.1 \mathrm{wpm}$

- Expert: $43.5 \mathrm{wpm}$

As with the ABC layout, we indicate 'greater than' $9.1 \mathrm{wpm}$ for the novice prediction since the letters are sequenced alphabetically. Although the expert figure is comparable to Qwerty, bear in mind that for unconstrained text entry, the method's utility hinges on the disambiguating algorithm's ability to provide reasonably accurate results. Implementation details are also extremely important, such as providing a mechanism to select from alternate plausible words (e.g. $228=$ 'bat' or 'cat'), or to explicitly enter a word when none is found in the dictionary.

2.4.2. With explicit entry: Other text entry techniques for telephone keyboards adopt some mechanism to explicitly identify each letter. Two schemes are (a) press each key one, two, three, or four times to select the 1st, 2nd, 3rd or 4th letter on the key, or (b) press a key followed by the number $1,2,3$, or 4 to select the 1 st, 2nd, 3rd, or 4th letter on the key. We choose the latter technique to predict with our model. So, for example, the name 'Jones' would be entered as the following sequence of numbers:

$\begin{array}{llllllllll}\mathrm{J} & & \mathrm{O} & & \mathrm{N} & & \mathrm{E} & & \mathrm{S} & \\ 5 & 1 & 6 & 3 & 6 & 2 & 3 & 2 & 7 & 3\end{array}$

Our predictions are as follows:

- Novice: $>7.4 \mathrm{wpm}$

- Expert: $22.6 \mathrm{wpm}$

The comparatively low predictions are, of course, due to the need to tap two keys for each letter entered.

\subsection{Just Type keyboard}

As with the telephone keyboard, the JustType keyboard ${ }^{4}$ places more than one letter on each key. The 26 letters of the alphabet are encoded on nine keys, with eight keys encoding three letters each, and one key encoding two letters (see figure 5).

The JustType keyboard works with a large dictionary and a disambiguating algorithm to determine the user's intended words. Unlike the telephone keyboard, the letter groupings are distinctly nonsequential. The groupings in figure 5 were chosen to optimize the performance of the disambiguating algorithm. ${ }^{5}$ After inputting the key coordinates in our spreadsheet model, we arrived at the following predictions: 


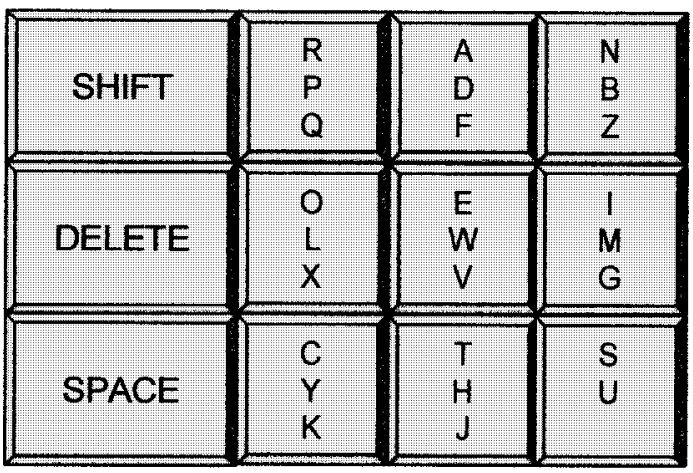

Figure 5. The JustType layout.

- Novice: 9.8 wpm

- Expert: $44.2 \mathrm{wpm}$

The expert entry rate is just $2.3 \%$ faster than for the Qwerty keyboard.

\section{Evaluating novice behaviour}

In most evaluations with input devices or interaction techniques, learning effects are considered a confounding factor. There are two common ways to deal with this. The first is to use a very primitive interaction taskone that quickly yields a high level of proficiency (e.g. MacKenzie et al. 1991). The second is to sufficiently practice subjects until a criterion level of performance is attained (e.g. Card et al. 1978). Both methods are pragmatic. Indeed, expert behaviour is not a 'state' that one captures, because human performance improves indefinitely with practice in both complex and simple tasks (De Jong 1957). ${ }^{6}$

Measuring novice behaviour, however, presents a different problem. The goal is to capture a snapshot of human performance at the onset of learning. We call this the 'novice experience'. This is particularly worthwhile for user interfaces targeted at consumers, since 'immediate' usability is important. New methodologies must be devised, because substantial learning may occur within a few minutes of exposure, and this effectively 'contaminates' the novice status of subjects.

In an earlier study, we captured the novice experience with the Graffiti alphabet of unistroke symbols for handwriting recognition (MacKenzie and Zhang 1997). We measured subjects' performance following oneminute and then five minutes of controlled exposure to the technology. In the following section, we describe a simple methodology, seeking to similarly capture the novice experience with various soft keyboards. We refer to our method as a 'quick test' rather than a 'novice test', because of the simple technique employed, and because of the special problem presented by layouts such as Qwerty where prior exposure is inevitable. Since fewer measures are gathered per subject, we used more subjects than are commonly employed in evaluations with input devices. (In Card et al's. 1978 study, for example, only five subjects were used.)

\section{Method}

\subsection{Subjects}

Twenty-four volunteer subjects (18 male, 6 female) were recruited from students and staff in our department at the University of Guelph. All were regular users of desktop computers with Qwerty keyboards. None had used pen-based computers previously on a regular basis.

\subsection{Apparatus}

We used a paper facsimile (similar to figures 1-5) for each of the following keyboards:

- Qwerty

- Dvorak

- $\mathrm{ABC}$

- Fitaly

- Telephone (with built-in disambiguation)

- JustType

Subjects sat at a desk with the keyboard layout in front of them on the surface of the desk. Text was entered by tapping on the paper image of the keyboard using a stylus. The stylus was borrowed from a Wacom graphics tablet. Prior to beginning, subjects were briefly shown the keyboard layout. The operation of each keyboard was explained to each subject. This was particularly important for the JustType and Telephone keyboards, since there were multiple characters per key.

\subsection{Procedure}

The following 45-character phrase of text was used:

the quick brown fox jumped over the lazy dogs

Each subject entered the phrase on each of the six keyboard layouts. The order of keyboards was counterbalanced using a $6 \times 6$ Latin square, with four subjects receiving each ordering. Since we sought to capture the novice experience, no practice trials were given and the 
text phrase was entered once only. We felt that this procedure, combined with data from 24 subjects, would provide stable measurements of the novice experience with each soft keyboard. The most serious flaw in this reasoning follows from the subjects' prior experience with Qwerty keyboards. We will say more on this later.

The total entry time was measured with a stop watch. Entry time (s) was converted to entry speed (wpm) as follows:

$$
\text { Entry_Speed }=\left(\frac{1}{\text { Entry }- \text { Time } / 44}\right) \times\left(\frac{60}{5}\right)
$$

The text phrase had 45 characters; however, since entry was timed from the first tap, there was no movement time to the first character. Therefore, entry time was divided by 44, rather than 45 . Taking the reciprocal transforms the measure into 'characters per second'. Multiplying by 60 and dividing by 5 transforms the measure into 'words per minute'.

Subjects were instructed to tap the phrase as quickly as possible while trying to avoid making mistakes. They were also reminded to tap spaces between words. As the experiment was a simulation of real soft keyboards and ran without data capture software, error rates were not recorded.

\section{Results and discussion}

The mean entry speed across all subjects and keyboards was $10.5 \mathrm{wpm}$. There was a significant effect of keyboard on entry time $\left(F_{5,23}=184.3, p<0.001\right)$. The Qwerty layout was the fastest $(20.2 \mathrm{wpm})$, while JustType layout was the slowest $(7.3 \mathrm{wpm})$. The results are summarized in table 1 . The relatively low standard deviations suggests that the mean scores for each layout were consistent across subjects.

That our test was a quick test rather than a novice test is evident in table 1 . Since all subjects were experienced desktop computer users, they were by no means 'novices' with the Qwerty layout. A similar-although less emphatic - statement can be made for the ABC and Telephone keyboards, since the sequential ordering of letters gives subjects a reasonable clue to each letter's location. Furthermore, over the duration of each test, the novice status fades as subjects become familiar with the arrangement of keys.

It is instructive to compare the observations from our quick test with the novice and expert predictions presented earlier. Since the observed rates should fall between the novice and expert predicted rates, they are presented in figure 6 as the middle of three bars for each keyboard.

Table 1. Entry speed vs. keyboard layout.

\begin{tabular}{|c|c|c|}
\hline \multirow[b]{2}{*}{ Keyboard layout } & \multicolumn{2}{|c|}{ Entry speed $(\mathrm{wpm})^{\mathrm{a}}$} \\
\hline & Mean & Std. Dev. \\
\hline Qwerty & 20.2 & 4.9 \\
\hline Dvorak & 8.5 & 2.0 \\
\hline $\mathrm{ABC}$ & 10.6 & 1.7 \\
\hline Fitaly & 8.2 & 2.2 \\
\hline Telephone & 8.1 & 1.9 \\
\hline JustType & 7.3 & 1.5 \\
\hline Mean & 10.5 & - \\
\hline
\end{tabular}

${ }^{a} n=24$ subjects.

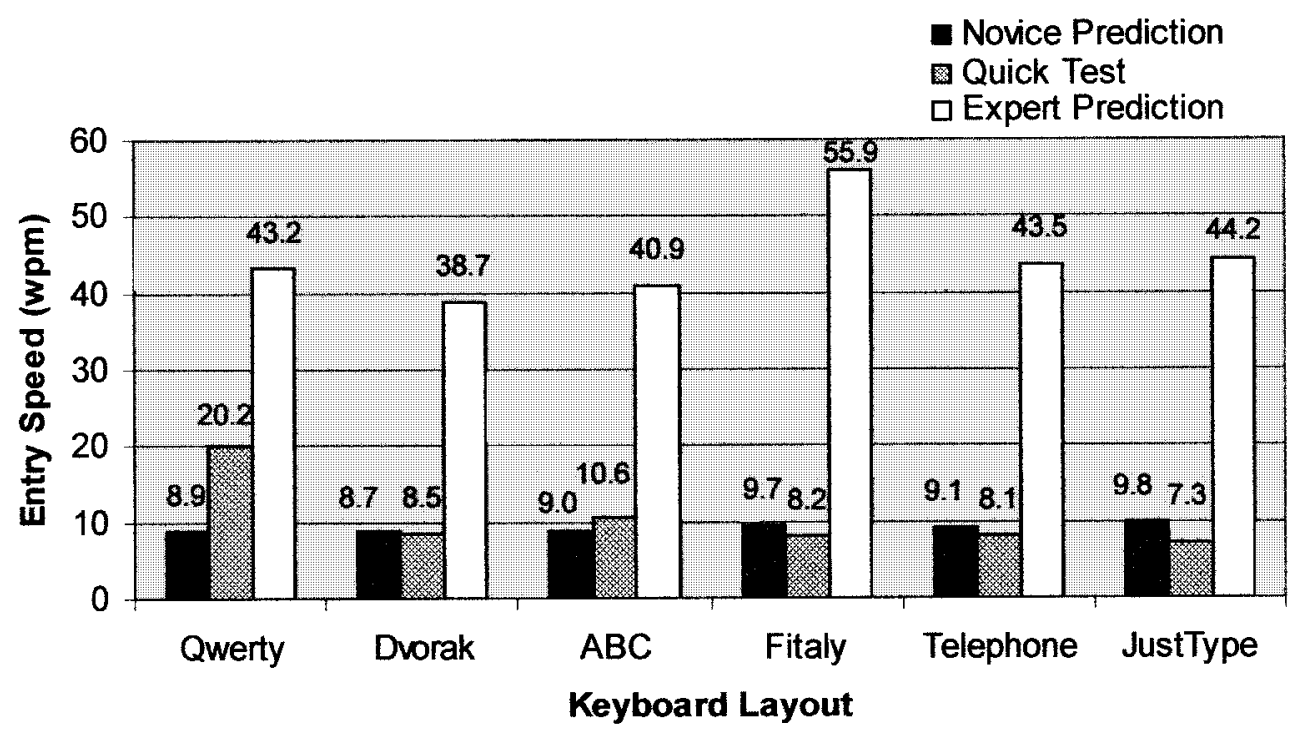

Figure 6. Comparison of novice and expert predictions and quick test observations for six soft keyboard layouts. 
At 20.2 wpm, our quick test measurement for the Qwerty layout illustrates subjects' familiarity with this keyboard. This figure is only slightly below the $22.9 \mathrm{wpm}$ figure measured in a test with a real Qwerty soft keyboard (MacKenzie et al. 1994), and is $46.8 \%$ of the predicted expert rate of $43.2 \mathrm{wpm}$.

The ABC layout, with its familiar arrangement of keys and with the large space bar, did not score as high as expected. The average entry speed of $10.6 \mathrm{wpm}$ is about half that of the Qwerty layout, and is only 17.8\% higher than the novice prediction of $9.0 \mathrm{wpm}$. This implies that subjects' daily experience with a Qwerty keyboard is an important advantage for the stylus tapping task.

We were surprised at the scores for the other four keyboard layouts. Ranging from 7.3 wpm (JustType) to $8.5 \mathrm{wpm}$ (Dvorak), all rates were below those predicted for novices. This is problematic since, as noted earlier, the novice predictions should be a lower threshold from which subjects rise quickly when working with a new keyboard layout. We have considered several reasons for the low entry rates. First, our text phrase included every letter of the alphabet. On the one hand, this is good because it ensures subjects visit every key during the task. On the other hand, the appearance of all 26 letters of the alphabet in a 45-character phrase means the phrase is not typical of common English. Thinking this might push the observed speeds down, we generated for each keyboard a novice prediction for the specific phrase, 'the quick brown fox .... These predictions differed very little from the novice predictions in figure 6 (typically $0.2 \mathrm{wpm}$ ), so we ruled out this explanation. Other explanations are explored in the next section.

\subsection{The novice experience}

Although the predictions are less striking for novices than experts, it is the novice experience that often determines the overall acceptance of new technology. Hence, it is important to capture aspects of the task that affect or determine novice performance.

\subsubsection{Visual scan time: For a soft keyboard with an} unfamiliar layout, the visual scan time is one aspect of the novice experience that must be examined. Our figure of $951 \mathrm{~ms}$ is the visual scan time for 27 choices, predicted using Equation 3. This estimate is sensitive to the slope coefficient in Equation 3, which we set to $200 \mathrm{~ms} /$ bit (Hick 1952). However, in Welford's extensive review of choice reaction time studies (1968: 60-104), slopes vary from about $160 \mathrm{~ms} /$ bit to about $320 \mathrm{~ms} / \mathrm{bit}$. Using Equation 3, this implies that the visual scan time could range from about $760 \mathrm{~ms}$ to about $1.52 \mathrm{~s}$ there- fore, one explanation for the low observed entry rates for the four 'novice' keyboards (figure 6) is that our $951 \mathrm{~ms}$ estimate of visual scan time is too low. Since the predicted vs. observed discrepancies range from $2.3 \%$ (Dvorak) to $28.6 \%$ (JustType), it is not clear how much of an adjustment may be warranted. It is our feeling, however, that other, more significant factors are at play, as discussed in the next section.

\subsubsection{Movement time: Other possibilities for the dis-} crepancies between our observations and the novice predictions arise from a re-examination of the movement component of the task. Our model assumes that text entry consists of a visual scan time- set to zero for experts-followed by a movement, with each movement beginning where the previous movement ended. This may be too simplistic. Since soft keyboards lack kinesthetic and tactile feedback, ongoing visual feedback is required, even for experts. Consider, as an example, a right-handed expert subject. Following a tap on the left side of the keyboard, much of the keyboard is obscured by the hand. If the following key is on the right, movement will proceed in that direction and the spatial arrangement of keys will be revealed as the hand moves toward the key. There may be a slight degradation in performance-one that is not accounted for in our model.

For novice subjects, the situation is quite different. We observed a definite pattern of lifting the hand above the keyboard after each tap. This behaviour was not limited to situations where the previous tap obscured the keyboard; it was a pattern that occurred consistently following each tap. Since novice subjects do not know the location of the next key, lifting the hand to a neutral, revealing position above the keyboard is a logical strategy. This is likely a parallel action, overlapping the visual search. Clearly, the motor component of the prediction is affected and this weakens our model's predictive power for novices.

Another limitation pertains to the size of the keyboard. According to our model a keyboard can be scaled up or down without affecting the movement time prediction. This is due to a simple characteristic in Fitts' law: If the movement distance is doubled (or halved) but target width is also doubled (or halved), the predicted movement time remains the same (see Equation 2). In other words, a keyboard may be scaled up or down without affecting the movement component of the model. Although this may be true within limits, it is probably not true in the extreme or when other identifiable factors enter into the task. There are a variety of such factors. For very small keys, the contact area of the stylus tip may be an appreciable percentage of the size of the keys. This would tend to reduce the 
effective area of the key. Drury and Hoffmann (1992) showed that an optimal data-entry keyboard will have an inter-key gap equal to the width of the data entry probe (viz., stylus tip). For very small soft keyboards, this effect may be important. This is likely confounded with parallax - the visual gap between the stylus' contact point and the visual feedback when the display is viewed at an angle. However, further study is needed before the effect can be quantified.

Another factor is the required limbs and muscles groups for the movement. For a small keyboard, the majority of the moves occur with the wrist anchored; whereas for larger keyboards the wrist is airborne as movement engages the forearm as well as the wrist. The highly learned task of handwriting is performed primarily with the wrist anchored.

\section{Conclusions}

Text entry on small mobile systems remains a challenge for computing systems of the future. Stylus tapping on a soft keyboard offers easy entry; however, rates are moderate at best and a keyboard must be presented on the systems' display, thus occupying screen real estate. Expert entry rates may reach $43 \mathrm{wpm}$ for the Qwerty layout, or in excess of $50 \mathrm{wpm}$ for optimized layouts. Because eye fixation is a requirement of interaction with soft keyboards, fatigue may prove a factor with prolonged use.

Novice entry rates are in the 7-10 word per minute rate for most layout permutations. However, experienced users of desktop computers may enter text with an immediate rate of about 21 words per minute on a soft keyboard with a Qwerty layout. This suggests that the venerable Qwerty layout is here to stay, both for physical keyboards on desktop computers and for soft keyboards that support stylus tapping.

\section{Notes}

${ }^{1}$ Our digraph tables includes $27 \times 27=729$ entries $(26$ letters plus the space bar), and is available in several formats via the World Wide Web. The URL is http:// snowhite.cis.uoguelph.ca/ will/bit95.tables.html.

${ }^{2}$ Such an experiment is described in the master's thesis by the second author. The experiment was a simple text entry task with the letter-to-key assignment randomized after each tap. The average entry rate for 12 subjects was 5.52 wpm (see Zhang 1998).

${ }^{3}$ Textware Solutions, 83 Cambridge St., Burlington, MA, 01803 USA, US Patent Number 5,487,616.

${ }^{4}$ Aiki Ltd., 219 First Ave. South, Suite 410, Seattle, WA, 98104-0680, USA, Patents pending.
${ }^{5}$ The layout in figure 5 is a 'sample' JustType keyboard, as provided by Aiki Ltd. Although the letter groupings are fixed, other key layouts are possible.

${ }^{6}$ If both human performance and practice are plotted in logarithmic scales, a continuous linear improvement in performance appears. That there is a diminishing return in the performance improvement vs. practice is inherently accommodated in the logarithmic transform of the measures.

\section{Acknowledgements}

We thank the members of the Input Research Group, at the University of Toronto and the University of Guelph for their assistance and suggestions. This research was supported by the Natural Sciences and Engineering Research Council (NSERC) of Canada, the University Research Incentive Fund (URIF) of the Province of Ontario, and Architel Systems Corp. of Toronto. We gratefully acknowledge these contributions without which this work would not be possible.

\section{References}

Blickenstorfer , C. H. 1996, Handwriting recognition is alive and well, Pen Computing Magazine, (August) 30-33.

Blickenstorfer, C. H. 1997, A new look at handwriting recognition. Pen Computing Magazine, (April) 76-81.

Card, S. K., English, W. K. and Burr, B. J. 1978, Evaluation of mouse, rate-controlled isometric joystick, step keys, and text keys for text selection on a CRT, Ergonomics, 21, 601 613.

De Jong, J. R. 1957, The effects of increasing skill on cycle time and its consequences for time standards, Ergonomics, 6, $51-60$.

Drury, C. G. and Hoffmann, E. R. 1992, A model for movement time on data-entry keyboards, Ergonomics, 35, $129-147$.

FITTS, P. M. 1954, The information capacity of the human motor system in controlling the amplitude of movement, Journal of Experimental Psychology, 47, 381 - 391.

Gentner, D. R. 1983, Keystroke timing in transcription typing, in W. E. Cooper (ed.), Cognitive aspects of skill typing, (Location: Springer-Verlag), 95-120.

Gopher, D. and RaIJ, D. 1988, Typing with a two-handed chord keyboard: Will the Qwerty become obsolete? IEEE Transactions on Systems, Man, and Cybernetics, 18, $601-$ 609.

Gould, J. D., Greene, S. L., Boies, S. J., Meluson, A. and RASAMnY, M. 1990, Using a touchscreen for simple tasks, Interacting with Computers, 2, 59-74.

Hick, W. E. 1952, On the rate of gain of information, Quarterly Journal of Experimental Psychology, 4, 11-26.

Hyman, R. 1953, Stimulus information as a determinant of reaction time, Journal of Experimental Psychology, 45, 188 196. 
Lewis, J. R., Potosnak, K. M., and Magyar, R. L. 1997, Keys and keyboards, in M. Helander, T. K. Landauer, and P. V. Prabhu (eds) Handbook of human-computer interaction, Chapter 54. (Amsterdam: North-Holland (Elsevier)).

MacKenzie, I. S. 1992, Fitts' law as a research and design tool in human-computer interaction, Human-Computer Interaction, 7, $91-139$.

MacKenzie, I. S., Nonnecke, B., McQueen, C., Riddersma, S. and Meltz, M. 1994, Alphanumeric entry on pen-based computers, International Journal of Human-Computer Studies, 41, $775-792$.

MacKenzie, I. S., Sellen, A. and Buxton, W. 1991, A comparison of input devices in elemental pointing and dragging tasks, Proceedings of the CHI '91 Conference on Human Factors in Computing Systems, 161-166.

MacKenzie, I. S. and Zhang, S. X. 1997, The immediate usability of graffiti, Proceedings of Graphics Interface '97, $120-137$.

Norman, D A. and Fisher, D. 1982, Why alphabetic keyboards are not easy to use: Keyboard layout doesn't much matter, Human Factors, 24, 509-519.
SEARS, A. 1991, Improving touchscreen keyboards: Design issues and a comparison with other devices, Interacting with Computers, 3, $252-269$.

Sears, A., Revis, D., Sawtski, J., Crittenden, R. and ShNeiderman, B. 1993, Investigation touchscreen typing: The effect of keyboard size on typing speed, Behaviour \& Information Technology, 12, 17-22.

Soukoreff, W. and MacKenzie, I. S., 1995, Theorectical upper and lower bounds on typing speed using a stylus and soft keyboard, Behaviour \& Information Technology, 14, $370-$ 379.

Welford, A. T. 1968, Fundamentals of skill. (London: Methuen).

Zhang, S. X. 1998, A high performance soft keyboard for mobile system. Master's Thesis, University Guelph. Guelph, Ontario, Canada. 